\title{
Axiomatization and Models of Scientific Theories
}

\author{
Décio Krause* Jonas R. B. Arenhart \\ Fernando T. F. Moraes \\ Research Group in Logic and Foundations \\ Department of Philosophy \\ Federal University of Santa Catarina
}

September 29, 2010

\begin{abstract}
In this paper we discuss two approaches to the axiomatization of scientific theories in the context of the so called semantic approach, according to which (roughly) a theory can be seen as a class of models. The two approaches are associated respectively to Suppes' and to da Costa and Chuaqui's works. We argue that theories can be developed both in a way more akin to the usual mathematical practice (Suppes), in an informal set theoretical environment, writing the set theoretical predicate in the language of set theory itself or, more rigorously (da Costa and Chuaqui), by employing formal languages that help us in writing the postulates to define a class of structures. Both approaches are called internal, for we work within a mathematical framework, here taken to be first-order ZFC. We contrast these approaches with an external one, here discussed briefly. We argue that each one has its strong and weak points, whose discussion is relevant for the philosophical foundations of science.

Keywords: Structures, Models, Set-Theoretical Predicates, Formal Languages.
\end{abstract}

\section{Introduction}

One of the central questions for those interested in the philosophical foundations of science is: what is a scientific theory? It is well known that the literature presents us with a cluster of different answers, but as a shortcut for the general wide idea, we might say that most philosophers of science nowadays employ in one way or another the word model to spell an answer. The widespread use of models in philosophical discussions - it appears not just in the characterization

*Partially supported by CNPq. 
of scientific theories, but also in discussions that go beyond this task - may be taken as tantamount to the great success of the so-called semantic approach to scientific theories in the present day philosophy of science. ${ }^{1}$ Starting in the middle $50 \mathrm{~s}$, this conception has been adopted by philosophers of different persuasions, and both realists and anti-realists have made use of it. Loosely speaking, a theory is seen as characterized by a class of models. But to simply state this is to say few. What are models? Models of what? How can we get them? In restricting the analysis to theoretical or abstract mathematical models, we may add: which kind of mathematics do we use to construct them (supposing that we do construct them)? Is this mathematics relevant for the construction of models in some sense? Are the models just first-order structures as those dealt with by standard Model Theory, as most philosophers seem to believe? These are questions that appear in between the lines of the discussions, but to which the literature gives us no clue. In this paper we intend to point to some of these problems and to discuss them.

\section{Models, models, and models}

Since models are central to the semantic approach, we must delineate its meaning at least for some basic discussion. The first point to acknowledge is that 'model' is not a so naïve term, for, taking a glimpse in the literature, we will be faced, for instance, with iconic models, analogy models, logical models, among other uses of the word (see for instance $[20, \S 2.1]$ for some examples). It is usually claimed that the semantic or model-theoretical approach to scientific theories was inspired somehow in the work of Alfred Tarski in the 1950s, when he started developing (standard) Model Theory, a branch of mathematical logic that deals mainly with the relations between first-order formal languages and structures that interpret them. So, as it is easily seen from the literature, for most philosophers of science (with a clear exception of Suppes), models of even scientific theories are models of first-order languages in the Tarskian sense, so we need to be careful in saying what they are, because, as we shall see, we can use models in a different sense than the Tarskian one. ${ }^{2}$ Really, we shall argue later that the models of the most relevant scientific theories are not always models in this Tarskian sense; in special, they are not first-order (or, as we prefer to call them, order-1) structures, in a sense to be defined below.

Despite being somehow historically accurate, this description is not the whole story, or at least it should not be. It is not simply the case that scientific theories are classes of structures in the sense of model theory, and that philosophers of science can employ the tools of this branch of mathematics to draw conclusions about it. Obviously, in the philosophical context of explaining what scientific

\footnotetext{
${ }^{1}$ Nowadays, the semantic approach is more a label that congregates a great number of philosophers of different orientations than a unified program of research; however, we maintain this name because it is widely used in the literature.

${ }^{2}$ As we shall see later, by model in a 'Tarskian sense' we mean a structure in which the non-logical symbols of a formal language are interpertred satisfying a set of sentences of this language and employing the corresponding notion of truth.
} 
theories are, when it comes to specify rigorously what we mean by models as well as the extent to which we can say a theory is a class of models, we are on slippy grounds. A first effort to bring this conception into a workable piece of philosophy stems from Patrick Suppes and his co-authors works, particularly with their axiomatization of particle mechanics. According to Suppes, to axiomatize a scientific theory is to present a set-theoretical predicate, namely, a formula of the language of (generally informal) set-theory. The set-theoretical structures that satisfy such a formula are called the models of the theory. That is, Suppes works within set theory; thus, we call this approach internal.

In this work we discuss how we can describe rigorously the idea that models somehow 'constitute' a theory. Based on Suppes works (here we mention [19, 20], where the reader can find additional references), a rigorous formulation of his basic idea was given by da Costa and Chuaqui [4], but in a sense this approach differs from Suppes, so we argue that one can understand the claim that a theory is a class of models in essentially two different ways, so as that each has its own advantages and its own costs. This is the kind of discussion one rarely finds in considerations about the meaning of the semantic approach, but that are recently coming gradually to light (see, for example, Muller [16]).

We begin, in the next section, with a general account of structures, and show how they can be developed inside ZFC set-theory. Since models in philosophical discussions are almost always set theoretical structures, the fact that we are adopting a particular theory of structures throughout lessens in nothing the generality of our work. Also, it must be remarked that even though there are alternative frameworks on which structures can be built, we follow here the standard approach and use ZFC.

\section{Structures}

Zermelo-Fraenkel set theory can be formalized in different ways. We can use a first-order logic as its underlying logic, with the language having as its only non-logical symbol a binary predicate $\in$. But we could also use a second-order language (or another higher-order one), as Zermelo preferred [15]. Of course these theories are not equivalent, and Zermelo's fear of non-standard models (which arise in first-order theories) is something which would deserve attention also from the point of view of the philosophy of science. Really, we don't know why philosophers of science prefer to suppose first-order set theory when they speak about the basis they are admitting. Anyway, as said above, we shall be working in first-order Zermelo-Fraenkel set theory with the Axiom of Choice, ZFC. ZFC without the axiom of choice will be termed ZF. As we shall discuss later, this choice of framework has its consequences.

The first definition we need is that of types:

Definition 3.1 The set $\tau$ of types is the least set satisfying the following conditions:

(i) $i \in \tau$ 
(ii) If $a_{0}, a_{1}, \ldots, a_{n-1} \in \tau$, then $\left\langle a_{0}, a_{1}, \ldots, a_{n-1}\right\rangle \in \tau, 1 \leq n<\omega$.

Here, $\omega$ stands for the set of natural numbers. We now define the notion of order of an element of $\tau$.

Definition 3.2 If $a \in \tau$, the order of $a$, denoted $\operatorname{ord}(a)$, is defined as follows:

(i) $\operatorname{ord}(i)=0$

(ii) $\operatorname{ord}\left(\left\langle a_{0}, a_{1}, \ldots, a_{n-1}\right\rangle\right)=\max \left\{\operatorname{ord}\left(a_{0}\right), \operatorname{ord}\left(a_{1}\right), \ldots, \operatorname{ord}\left(a_{n-1}\right)\right\}+1$.

In the next definition, the usual set-theoretical operations of power set and cartesian product are being used, and are denoted respectively by $\mathcal{P}$ and $\times$.

Definition 3.3 Given a non-empty set $D$, we define a function $t$, called scale based on D, having $\tau$ as its domain, as follows:

(i) $t(i)=D$

(ii) If $a_{0}, a_{1}, \ldots, a_{n-1} \in \tau$, then $t\left(\left\langle a_{0}, a_{1}, \ldots, a_{n-1}\right\rangle\right)=\mathcal{P}\left(t\left(a_{0}\right) \times t\left(a_{1}\right) \times \cdots \times\right.$ $\left.t\left(a_{n-1}\right)\right)$.

Thus, following the usual extensional set-theoretical definition that a relation is a set of ordered pairs, for each type $a$, the mapping $t$ gives us the set of all relations of that type, built from the elements of $D$. Obviously, as the orders of types increase, the objects attributed to them by $t$ increase in complexity too. We say that the elements of $t(a)$ have type $a$. For example, the elements of type $i$ are, by definition, the elements of $D$, and are called individuals. Then, $\langle i\rangle$ is the type of the properties of individuals, $\langle i, i\rangle$ is the type of the binary properties of individuals, $\langle\langle i\rangle\rangle$ is the type of the unary properties of unary properties of individuals, that is, sets of sets of individuals, and so on.

Definition 3.4 The scale based on $D$ is the set $\bigcup($ ranget $(D))$, denoted by $\varepsilon(D)$.

Definition 3.5 The cardinal $\kappa_{D}$ associated to $\varepsilon(D)$ is defined as

$$
\kappa_{D}=\sup \left\{|D|,|\mathcal{P}(D)|,\left|\mathcal{P}^{2}(D)\right|, \ldots\right\} .
$$

Definition 3.6 A structure $\mathfrak{A}$ based on $D$ is an ordered pair of the form

$$
\mathfrak{A}=\left\langle D, R_{\iota}\right\rangle
$$

where $R_{\iota}$ is a sequence of elements of $\varepsilon(D)$, and we suppose that the domain of this sequence has cardinal strictly less than $\kappa_{D}$. We say that $\kappa_{D}$ is the cardinal associated with $\mathfrak{A}$, and that $\varepsilon(D)$ is the scale associated with $\mathfrak{A}$. 


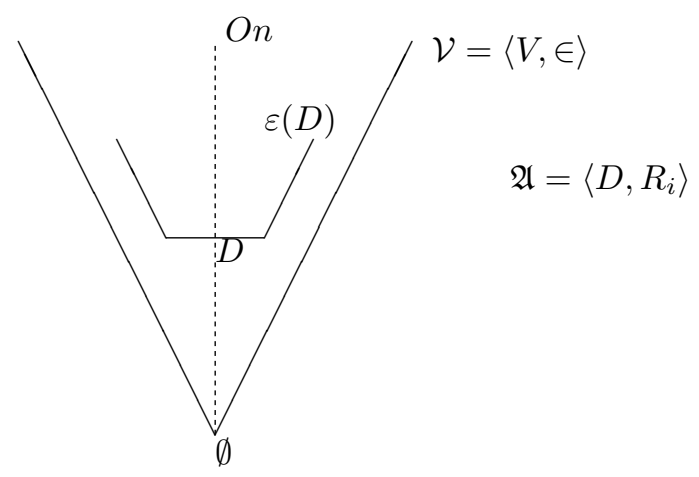

Figure 1: A scale $\varepsilon(D)$ built within the well-founded model of ZFC. For details, see the text.

As we said before, each element of $\varepsilon(D)$ (see Figure 1) has a certain type, for it belongs to $t(a)$ for some $a \in \tau$. Now, the order of a relation is defined as the order of its type. The order of $\mathfrak{A}$, denoted ord $(\mathfrak{A})$, is the order of the greatest of the types of the relations of the family $R_{\iota}$, if there is one, and if there is no such relation, we put $\operatorname{ord}(\mathfrak{A})=\omega$.

If a structure has order $\kappa$, we say that it is an order $-\kappa$ structure. Groups are examples of order -1 structures, for they contain only order- 1 relations (actually, depending on the formalization, they contain just one order-1 relation of type $\langle i, i, i\rangle$, for the binary operation on the domain can be seen as a ternary relation). ${ }^{3}$ More involved structures can contain several sets, divided up into principal and secondary sets [2, Chap.4]. For instance, vector spaces are structures of the form $\mathfrak{E}=\langle\mathcal{V}, F,+, \cdot\rangle$; here $\mathcal{V}$ is the set of vectors, $F$ is a field, + is a binary operation on $\mathcal{V}$ (addition of vectors) and $\cdot$ is the multiplication of a vector by an element of $F$, all of them obeying well-known axioms. Such structures can still be represented via the general form of definition 3.6, for the domain can be formulated as the union of all principal and secondary sets, with suitable adaptations in the definition of the relations in question. Typical examples of structures which are not order- 1 structures are the following ones: well-orders, ciclic groups, Dedekind-complete ordered fields [11, p.89], so as the most relevant structures of the empirical theories, as we shall see soon.

Structures and languages Another important concept is that of the language of a structure. The first-order language of ZFC will be denoted by $\mathcal{L}_{\epsilon}$, and we assume that it has only one non-logical symbol - namely, the binary predicate $\in$ - and no individual constants (although the latter can be considered in alternative formulations). Suppose we have a structure $\mathfrak{A}=\left\langle D, R_{\iota}\right\rangle$, defined in $\mathcal{L}_{\epsilon}$, whose domain $D$ may comprise several sets, and the relations

\footnotetext{
${ }^{3}$ If we denote the binary operation on a set $G$ by $\circ$, so that the image of the pair $\langle a, b\rangle$ is written $a \circ b$, then this binary operation can be treated as the relation $R=\{\langle a, b, a \circ b\rangle$ : $a, b, a \circ b \in G\}$.
} 
in $R_{\iota}$ are order- $n$ relations $(n \geq 1){ }^{4}$ Notice that the employed symbology makes use of symbols such as $\mathfrak{A},\langle\rangle, D, R,, \iota$, which do not belong to $\mathcal{L}_{\epsilon}$. Since $\mathcal{L}_{\in}$ does not contain individual constants, we have basically two alternative approaches to represent such objects in a (perhaps extended) language. The first one consists in introducing these symbols to abbreviate formulas of $\mathcal{L}_{\in}$ that define these concepts, so we needn't change $\mathcal{L}_{\in}$ (for instance, usually $x \subseteq y$ abbreviates $\forall z(z \in x \rightarrow z \in y))$. The second one corresponds to an enlargement of the language itself through the introduction of new constant symbols. Notice that in this second approach we enlarge the language $\mathcal{L}_{\in}$ with new symbols such as $G$ and $\circ$ in the case of groups (as we shall see below), and then a group structure can be built in this extended language (within ZFC). In the same vein, by adding $V, K,+, \cdot$ (among other symbols), vector spaces structures can be formulated as well. Each structure will have its primitive symbols, in the general setting encapsulated by the relations $R_{\iota}$.

However, as we shall se below, we need not only to speak about structures, but also of objects of the scale $\varepsilon(D)$ based on $D$. According to one of the approaches to scientific theories (discussed in section 7), we could use either the language of the theory of types or infinitary languages, and these languages can also be built with the resources of first-order ZFC, such as the languages $\mathcal{L}_{\mu \kappa}^{\eta}$, where $\mu, \kappa$ and $\eta$ are cardinals such that $\kappa \leq \mu$ and $1 \leq \eta \leq \omega$, and $\omega$ is the first infinite cardinal (see [5], [6]). In an infinitary language, we can form conjunctions and disjunctions of sets of formulas of cardinality less than $\mu$, and blocks of quantifiers of length less than $\kappa$. We then have first-order languages when $\eta=1$, second-order languages when $\eta=2$, and so on, until $\eta=\omega$, which, when also $\kappa=\mu=\omega$ is an order- $\omega$ language suitable for simple theory of types.

As we shall see from now on, there are two basic procedures to work within ZFC: in a first approach, we can 'model' a certain domain of knowledge by a certain mathematical structure, and then formulate the postulates of the corresponding theory in the language $\mathcal{L}_{\epsilon}$, perhaps by considering also additional symbols used to speak about the elements of the structure. Alternatively, we can formulate the postulates in a formal language for the structure, in a sense to be explained below (see section 7). This language can be, for example, some version of the language of theory of types or even an infinitary language. The postulates, in both versions, will have the structure as its model, but, as we shall discuss, the relation between postulates and structure is different in both approaches. In discussing the relation of postulates and structures satisfying them, we must consider the role of postulates in determining a class of models and how this can be achieved.

\footnotetext{
${ }^{4}$ We recall that the order of a relation is not necessarily the same as its arity. For example, a binary relation, that is, a member of $t(\langle i, i\rangle)$, is a relation of order 1 .
} 


\section{Internal and external axiomatizations}

It is usually agreed that the semantic conception of theories has an advantage over the syntactic approach (usually associated to the logical-positivistic philosophy) once it does not focus on language or, at least, language would be not the essential point to be emphasized; the semantic approach focus on models. But language cannot be completely ignored for, in order to group a class of structures, we must provide a set of postulates they model. In other words, we need to employ the axiomatic method, and this involves the stipulation of also language and deductive apparatus. To begin, we can say that the axiomatic basis of a theory $T$ encompasses three levels of postulates (below we shall discuss with more details this schema - see section 5; the proposals associated to the logical positivism, called the Received View, can be conformed to this schema-see [18, pp.50-51], [16, §2.1]):

(i) The logical postulates, say those of classical first-order logic with identity. But in the general setting, we could use also higher-order logics, infinitary languages or logics, or still other non-classical logics. In the case of the Received View, of course the logic would be elementary classical logic with identity, perhaps augmented by modal operators - [18, loc.cit.].

(ii) A group of 'mathematical' postulates, say those of first-order ZFC (these axioms would be absent in the Received View approach). Perhaps only a small part of ZFC is to be used, say ZC, the Zermelo set theory (but without ur-elements, although these entities may be interesting in empirical sciences to represent things that are not sets) plus the axiom of choice (in agreement with Muller [16, loc.cit.]).

(iii) The specific postulates, which depend on the particular field being analyzed. According to our full schema, these postulates would be sentences of the language of ZFC, perhaps enriched by additional concepts and terms referring to the supposed domain of investigation.

The notion of deduction is that of the employed logic, in general, in accordance (when no other logical symbols are introduced) with elementary logic, but now in the deductions we may have axioms of ZFC as premises, so the logic is not 'exactly' elementary, as is necessary for the consideration of higher-order theories and languages, typical of scientific theories. We can consider alternatives to (i) plus (ii), for instance, by supposing some higher-order logic (except for some limiting cases where they are not sufficiently strong) or even category theory. But the above schema is quite general for the discussions usually made in the philosophy of science. ${ }^{5}$

We have basically two alternatives to consider axioms (i)-(iii). The first we call the internal approach, and identify it with the semantic approach. This internal approach can be seen from at least two distinct points of view: the

\footnotetext{
${ }^{5}$ Let us insist that, although ZFC is based on a first-order language, its expressive power is greater than that of elementary theories.
} 
Suppes' approach (SA), and the da Costa \& Chuaqui's (CCA) approach. In considering each approach, we may turn to the axioms (iii). The second approach is called internal. Let us explain them briefly in what follows.

The Suppes approach Suppes' 'Model Revolution' (this expression is Muller's [16]) is analysed with details by Muller in his mentioned text. We do not wish to make a different proposal than Muller's, but just to emphazise certain aspects which even his important paper does not cover. Basically, Suppes' approach consists in describing a structure (truly, wide class of structures) which 'models' a certain scientific domain. It does not matter us here the details about how this can be done, something that in general involves expertise, scientific background, experimentation, insights, and other processes to cope with the phenomenal data. For instance, classical particle mechanics (Newton's style) is characterized by a structure such as $\mathfrak{P}=\langle P, T, m, s, f\rangle$ (as we shall see below), while groups are given by a structure of the form $\mathfrak{G}=\langle G, \circ\rangle$, or $\mathfrak{G}=\left\langle G, \circ, e,^{-1}\right\rangle$ depending on the axioms we use, etc. Then we write down the postulates of the theory of that domain in the language $\mathcal{L}_{\epsilon}$, possibly enriched with additional symbols and concepts. These additional symbols can be introduced by different means, for example, as abbreviations of formulas of $\mathcal{L}_{\in}$ in the metalanguage, ${ }^{6}$ or the we introduce the new symbols in the language through some theory of definition (basically, obeying Leśniewski's conditions [19, Chap.8]). When we say that the given structure 'models' a certain field of knowledge, we roughly understand that the scientist, when interested in investigating some domain, starts from choosing, yet sometimes not consciously, certain concepts and properties/relations/operations that may hold for the objects under investigation. It is of course an abstraction to suppose that all of this can be described by presenting a mathematical structure, but this generalization fits what happens in a quite general form. Then specific postulates (of level (iii) above) are supplied for defining the theory of that structure (or of that field). These axioms can be abbreviated by means of a set-theoretical predicate, a formula of the language of ZFC (perhaps extended) which stand for the conjunction of the axioms; in the next section we give some additional details. In some cases, the scientist is defining a species of structure in the sense of Bourbaki [2, Chap.4], as we shall see in section $6 .^{7}$

What about axioms (i) and (ii)? They are subsumed by the ZFC plus underlying logic postulates and need not to be mentioned. Thus, as we have already referred to above, the notion of deduction is that of ZFC, hence ZFCpostulates may enter in the deductions, so the achieved theory, strictly speaking, is not elementary (Muller doesn't mention this important point). And there is another important point also not mentioned in general (neither by Muller), which is the following. The given structure would be a model of the given axiomatization in a sense. But, in what sense? Really, the word model must be

\footnotetext{
${ }^{6}$ As we do when use $x \subseteq y$ to abbreviate $\forall z(z \in x \leftrightarrow z \in y)$.

${ }^{7} \mathrm{Da}$ Costa and Chuaqui have identified their version of Suppes' set-theoretical predicates — see below— with Bourbaki's species of structures [4].
} 
dealt with here with care: it is a characteristic of this approach that in order to grant that a structure satisfies the postulates, we need to prove that the objects and relations of the structure have the properties stated in the postulates. We do it in ZFC. For instance, in order to prove that the structure $\mathcal{R}=\left\langle\mathbb{R}^{n}, \mathbb{R},+, \cdot\right\rangle$ models the postulates of vector space, we prove as a theorem of ZFC that

$$
\left\langle\mathbb{R}^{n}, \mathbb{R},+, \cdot\right\rangle \models \text { (vector space axioms), }
$$

where '(vector space axioms)' stands for the translation to an adequate language (built in ZFC) of the postulates for vector spaces. As it is easy to note, this is how we proceed in the standard mathematical practice.

What then is a theory? As we have seen, a structure within ZFC (or within a model of ZFC) encompasses elements of the scale $\varepsilon(D)$, where $D$ comprises the domains under consideration (cf. section 3). As a set, the structure 'lives' in a certain $V_{\alpha}$ of the cumulative hierarchy for some adequate ordinal $\alpha$ (for we are supposing here that the Axiom of Foundation holds); thus, by adapting Muller's good terminology, a theory $T$ may be written as

$$
T=\left\{\mathfrak{A} \in V_{\alpha}: \mathrm{P}(\mathfrak{A})\right\},
$$

where $\mathrm{P}$ is the set-theoretical predicate that axiomatizes $T$ (see the next section). Thus, a theory is a class of models (in general, $\left\{\mathfrak{A} \in V_{\alpha}: \mathrm{P}(\mathfrak{A})\right\}$ is not a set of ZFC), the models of the set-theoretical predicate or, alternatively, of the axioms of the theory.

As an example, consider again the case of classical particle mechanics (CPM) in Newtonian style. As we have seen, according to McKinsey et al., we can 'model' a classical system of particle mechanics by a 5 -tuple $\mathfrak{P}=\langle P, T, m, s, f\rangle$ where $P$ is a non-empty set (the 'particles'), $T$ is a set of real numbers (say expressing an interval of time), $m$ is a function from $P$ to $\mathbb{R}^{+}$so that if $p \in P$, then $m(p)$ is the mass of $p, s$ is a function from $P \times T$ in $\mathbb{R}^{3}$ (really, their original approach [14] can be described with more generality; see [20, Chap.7]), so that $s(p, t)$ is a vector expressing the position of the particle $p$ at time $t, f$ is a function with domain $P \times T \times I$, where $I$ is a set of positive integers, so that $f(p, t, i)$ is a vector representing the forces acting on $p$ at $t$. All these concepts are subjected to Kynematical and Dynamical axioms (written in the ZFC language suitably enriched) which do not concern us here [19, Chap.12], [20, Chap.7], so that we may suppose as well defined the corresponding set-theoretical predicate. It is not difficult to find other models for these postulates; in fact, the postulates define a class of models, as we have seen. Even if we do not present them here, it is easy to acknowledge that most of them may be just mathematical structures without having necessarily any connection with physics. But other models can fit (as we believe) parts of certain fields in physics. The models of $\mathrm{CPM}$ are structures like $\mathfrak{P}$, and are set-theoretical constructs, really structures within ZFC.

The da Costa-Chuaqui approach The da Costa-Chuaqui (CCA) approach also starts with a structure, given as before, but now we look for a formal 
language for that structure (in the sense of section 3), and in this language, we formulate suitable axioms which will have the structure as a model in the Tarskian standard sense. Note that here we look for models, although they are not in general order-1 structures, so as the languages are in general not firstorder languages. It is a characteristic of this approach that different languages may be used for this task, each with its costs and benefits, for there are infinitely many formal languages that could be used as suitable for the given structure [5]. In this case, as in the previous one, we are working within a mathematical framework, again the first-order ZFC for example. So, we may also leave implicit the postulates (i) and (ii), and just present those of level (iii), but now formulated in the formal language of the structure (in section 7 we shall see an example of one of such languages). Important to realize that this language is not the language of ZFC, so we are not modeling its axioms; consequently, we may look for models build in ZFC proper, which could not be done if the postulates of ZFC were being axiomatized too (supposing ZFC consistent of course). In presenting the postulates (iii), sometimes we need to enrich the language of ZFC, and in this case we proceed according to one of both ways mentioned before (that is, either as abbreviations in the metalanguage or by Leśniewski's criteria).

The main differences between Suppes and da Costa-Chuaqui approaches is that, now, the axioms are not formulated in the first-order extended language of ZFC, but we may consider even higher-order or infinitary languages, as we shall see in section 7. Thus, the models are, as the most interesting cases show, not order-1 structures (by the way, as in Suppes' approach too), so their general theory is not described by standard Model Theory. Da Costa and Chuaqui introduced the concept of Suppes predicate in their paper, and this concept is similar, but not exactly identical, to the set-theoretical predicates used by Suppes. In order to clarify this point, we need to consider other details of each of the two proposed above approaches, but now, for the sake of coherence, let us comment in brief on the 'external approach'.

\section{The external approach}

Now, we discuss the second alternative to axiomatization in continuation to what was said in the beginnings of section 4 , although this approach does not play a fundamental role in this paper. Let us call it the external approach or the syntactic approach. Here, as well characterized by K. Kunen [11, pp.28ff, $186 \mathrm{ff}]$, we firstly work in an intuitive metatheory, which must be adequate for we to express some basic things, such as the understanding that the set $\{1,2\}$ has two elements, what is an infinite sequence of numbers, what is a relation, and so on. Beginning with such a finitistic reasoning, gradually we erect suitable languages to cope with the axioms (i)-(iii) of section 4, until we reach to formal theories, such as ZFC. In particular, we may arrive at the formulation defended by the logical positivism, as discussed in F. Suppe's article at [18] and in [16, $\S 2]$. Then, suppose we have got ZFC set theory. Now we 'enter' ZFC and start 
all again, but formally. As Kunen says, "logic [and of course this applies to other theories] must be developed twice" [11, p.191].

Thus, the relevant concepts, such as those of relation and function will have two representations, one in the informal metatheory (which of course can also be formalized at the expenses of using an informal meta-metatheory, and so on), and another in the formal theory. As it is clear, not always the concepts agree in both formulations. For instance, the intuitive set of natural numbers may have a completely different corresponding set in a given model of formal first-order arithmetics (see [10]). In this case, we need to list the postulates (i), (ii) and (iii), and we do not ask for models, but just derive consequences of these postulates, which we (informally) interpret as telling us things about the field under analysis. Important to acknowledge that, according to the standards, here we do not look for models, but for proofs. In mathematics, this is the approach of Bourbaki. To him, to do mathematics is to write formulas in a paper according to certain rules he presents us in his book on set-theory [2]. In physics, Ludwig's approach to quantum mechanics runs quite similarly, based on Bourbaki's species of structures, in a purely syntactic way [12]. Interesting as this approach can be, it demands a great appeal to the underlying language, logic, and mathematics, and does not fit the pragmatic views of the scientists, who in general are not occupied with logic and set theory. By the way, this is the procedure we follow when using either a non-classical logic or a non-classical (for instance, a non-cantorian) set theory. ${ }^{8}$ There is no semantics, neither in the sense of Suppes discussed before, nor in the standard sense of Tarski.

According to this way of dealing with the matter, the concept of truth is syntactical; a certain proposition is true if we find a proof for it, and it is false if its negation has a proof. Of course there is no violation of classical logic, for the excluded middle law continues to hold in the theory, although it may be not valid in the metatheory. Thus, although Bourbaki's mathematics is classic, its metamathematics is not, being constructive in a certain sense. In fact, if we try to find a model also for the axioms (i) and (ii), we need to model the membership relation $(\in)$, and so we are modeling not only the specific theory itself (axioms (iii)), but also logic and set theory (ZFC), and a model of such a theory cannot be obtained in ZFC proper (supposed consistent), due to Gödel's second incompleteness theorem, as it is well-known. An alternative would be to work with a stronger theory, such as ZFC plus universes [3], but we shall not touch this point here. ${ }^{9}$

\footnotetext{
${ }^{8}$ Non-cantorian set theories (the terminology is Cohen's), roughly speaking, depart from standard set theories for incorporating a form of negation of the axiom of choice.

${ }^{9}$ In a theory with universes, we can prove the consistency of ZFC, in particular to find models of ZFC.
} 


\section{Theories as models, part I: Suppes approach revisited}

Let us go back to the semantic approach. With structures at hands, 'modeling' some field of knowledge, how can we go to interesting scientific theories? If theories are characterized as some kind of class of models, then we should make sure that there is some way to group all the relevant models somehow. In his works ([19, chap. 12], [20] among several others), as we have seen, Patrick Suppes discussed and presented some examples of how to axiomatize scientific theories using set-theoretical predicates, that is, he put forward a way of characterizing classes of structures satisfying a set-theoretical predicate whose main purpose was to lay some constraints on the kind of allowed structures. However, he did not provide a rigorous definition of how a set-theoretical predicate should be written, hoping that the intuitive idea behind his proposal would be clear from the given examples. As we mentioned before, it is debatable whether Suppes advocates the semantic approach (as he himself provocatively says, answering a claim in this sense posed by Muller - see [21]), but his works on the topic have inspired many reflections by the defenders of the semantic approach, and his program has sometimes been seen as defending this point of view (again, see the interesting discussion in [16]). In fact, as we are arguing in this paper, it is the very idea of semantic approach that is needing some clarification.

Suppes says he assumes intuitive set-theory, but we can continue to suppose that our discussion is conduced inside ZFC, at an informal level, that is, without justifying the constructions. The first immediate advantage of starting with ZFC (or with some suitable set theory) is that one can employ all of the mathematics needed without explicitly having to axiomatize or listing its suppositions, for 'everything' we need is by hypothesis already built inside ZFC. Also, one leaves metamathematical considerations of usual axiomatizations, which proceed through the elaboration of formal systems, and works in the known mathematical environment, simply by making use of the mathematics at disposal. This last point was one of the attractions of the semantic approach over the Received View (see a full discussion in [18]), which proceeded through formal systems, but we shall not make further comments on this point here; what we have already said suffices for our purposes.

In order to be more precise and pay some debts from above, let us see how we can define a set-theoretical predicate for groups. Suppes gives various alternative formulations of this theory (mainly due to his concerns with the theory of definition and the search for an adequate and perspicuous form which conforms some rigorous standards of definition). To fix ourselves in one of the proposed definitions, we can define a group through the following set-theoretical predicate, given informally first: a group is an algebra $\mathfrak{G}=\langle G, \circ\rangle$ where $G \neq \emptyset$ and $\circ$ is a binary operation on $G$, and ..., the dots being completed by the usual group axioms, written in the language of ZFC. Now, this is an example of a set theoretical predicate for group theory and, as Suppes claims, it is entirely in conformity with the usual mathematical practice. 
To be more precise, and using some terminology introduced before, we could specify the nature of the elements of the structure as well. So, a group $\mathfrak{G}$ is an ordered pair $\langle G, \circ\rangle$ with $\circ \in t(\langle i, i, i\rangle)$ satisfying $A 1, A 2$ and $A 3$ (where the $A$ s are the usual group axioms, namely, the associativity of $\circ$, the existence of a neutral element, and the existence of inverses). In symbols, the predicate $\mathfrak{G}$ is (or maybe) the following one:

$$
\mathrm{P}(\mathfrak{G}) \leftrightarrow \exists G \exists \circ(\mathfrak{G}=\langle G, \circ\rangle \wedge G \neq \emptyset \wedge \circ \in \mathcal{P}(G \times G \times G) \wedge A 1 \wedge A 2 \wedge A 3)
$$

Suppes calls a group any structure $\mathfrak{G}=\langle G, 0\rangle$ that satisfies this predicate. Nothing specific is said about the satisfaction relation, or about the language in which the axioms are formulated. But, as we mentioned before, it is the enlarged language of the set theory (with symbols such as $\mathfrak{G}, \circ$, etc.) which is being employed, and this is in conformity with the standard use in mathematics. How does one show that some structure is in the class characterized by the predicate so defined? For example, how does one show that $\langle\mathbb{Z},+\rangle$ is a group? One simply derives, as theorems of ZFC, that the elements of $\mathbb{Z}$, along with the operation + , have the properties required for something to be a group. Then, $\langle\mathbb{Z},+\rangle$ is said to satisfy the axioms for group theory and, so, the structure is a model of group theory, or it is a group.

Now, one should notice that the words 'satisfaction' and 'model' have very different senses from the ones logicians usually attribute to them. Really, there is no formal language being interpreted in some structure, no interpretation function attributing 'adequate' items of the structure to linguistic items, and no constraints on the formulation of the axioms. In the standard Tarskian sense, models are structures that satisfy (in a well-known specified sense) some sentences in a specified language. What one really does, in Suppes approach, restricting ourselves to the particular case we are discussing, is to show, for example, that $\vdash_{Z F C} \forall x, y, z \in \mathbb{Z}(x+(y+z)=(x+y)+z)$, and the same holds for the other axioms. That is the idea behind Suppes' suggestion that philosophy of science should work with mathematics, not with metamathematics (see, for example, [20]). This way, we are again agreeing with Muller's analysis (cf. § 6 of his paper). So, seen this way, a set theoretical predicate in the sense of Suppes characterizes a class of structures, right, but it looks difficult to take them in the usual sense of the logician (that is, as structures satisfying the axioms). Furthermore, since Suppes attributes special role to isomorphisms and representation theorems, it seems reasonable that he should follow this kind of approach, focusing on these notions and not paying attention to the usual worries of logicians with topics as consequence relations, definability and conditions on the applicability of model-theoretical tools.

To emphasize this point a little bit more, we emphasize that we are taking seriously the idea that one should employ mathematics, not metamathematics in these analyses. In this sense, what we are calling axiomatization in the sense of Suppes differs from axiomatization in the logicians' sense (or in da Costa-Chuqui approach). As we mentioned, in this case we are dealing with the mathematical objects themselves, that is, since $\mathbb{Z}$ is a term in the language of set theory, it 
denotes the set of integers (in some construction in ZFC). When we say that the structure composed by this set with the operation + (which is also a term in the language of set theory, denoting an operation on $\mathbb{Z}$ ) have some property, we state this directly in the language of ZFC, and we do not announce the property first in a formal language, then coordinate the symbols of the language with these sets and then proceed to show that the formula expressing the property is satisfied by the structure $\langle\mathbb{Z},+\rangle$. This would involve a shifting to metamathematics. In the Suppesian approach, as we read it, though, there is no such shifting, and we are always in mathematics, speaking about the set theoretical items directly, without the mediation of formal languages. Maybe this kind of argumentation does not make justice to the way Suppes himself understands his work, for he seems to be committed with the Tarskian concept of model, but we thing that our interpretation is a clear account of what he in fact does (in this respect, we entirely agree with the analysis of Muller [16]).

Taking models of ZFC into account An important remark is in need here. From a metatheoretical point of view, the class of models 'selected' by a predicate will obviously be dependent on the model of ZFC we are working in, and this may be important in certain situations. Just to exemplify what we mean, suppose that our theory makes use of the set $A=\left\{2^{\aleph_{0}}, \aleph_{\alpha}\right\}$, where $\alpha$ is a cardinal. How many elements has this set? It depends on the model of ZFC we are considering. Since the notion of cardinality is not absolute -it varies from model to model - we may have situations where $A$ has two elements, say when $\alpha=\aleph_{\omega}$, but in other models $A$ may have just one element, say when the continuum hypothesis holds and $\alpha=1$-see [11, pp. 67, 160]. Furthermore, since ZFC is a first-order theory, if consistent, its models cannot be built in ZFC proper. In particular, if consistent, the first-order ZFC has a denumerable model, and for instance the real numbers in this model are countable. This fact may have interesting or even disastrous consequences for the philosophy of science when we go to the next step and try to relate the theory with the experiments. Really, most physical theories use real numbers, but they (so we suppose) think of them as being the (intuitive) 'full' real numbers system. What happens if they are not this set but a denumerable one? Questions like these are of curse not side questionings, but should be also be considered seriously. Furthermore, as Muller correctly said, and Suppes agrees, Suppes does not speak of the Being, for as we have remarked, he is most occupied with other sides of theories, but the question is also relevant. In a nutshell: what can we say about the empirical theories when we consider a denumerable model of ZFC? Can we study scientific theories trying to capture empirical facts of the world in such a model? What does the world looks like according to a theory as a set of structures in this particular model? In fact, what are the consequences of, for instance, countable models for some of the most well-known philosophical frameworks built inside the semantical approach? How would it affect, for example, the empirical substructures of the models of empirical theories in van Fraassen's account? Or, how would it affect the data models in 
Suppes' account? We point to these topics as interesting questions deserving further elucidations, but we shall not address to them in this paper.

Anyway, this last topic deserves a further final comment. Along with the idea that the tool for the philosopher should be mathematics, not metamathematics, some considerations immediately come into play. First of all, if by mathematics in this case we do not mean 'to work inside ZFC', then one could employ different set theories to do the required job. Using some weaker form of the axiom of choice, for example, we could try to use some kind of non-cantorian set theory, in which some of the usual results of classical mathematics do not hold. This was partly mentioned when we introduced the axioms (i), (ii) and (iii) above. For instance, take 'Solovay's set theory', that is, ZF+DC+Solovay Axiom, where $\mathrm{ZF}$ is the theory ZFC without the full axiom of choice, DC is the axiom of dependent choices, and Solovay Axiom is a proposition that states that every set of real numbers if Lebesgue measurable (the details do not matter us here). In this theory, we can prove that all operators on a Hilbert space are bounded [13]. Thus, how could we use the standard formalism of non-relativist quantum mechanics (QM) in such a framework, since in QM we need unbounded operators (for instance, position and momentum)? Of course we would be in trouble.

Sure that we could also employ some very different set theory, other than ZFC, say Quine-Rosser's NF, some paraconsistent set theory, fuzzy set theory or quasi-set theory instead of ZFC (NF and fuzzy set theory are supposed to be known; for paraconsistent set theory, see [7]; quasi-set theory can be seen in [8]). Maybe for some purposes it would be very interesting to check how some specific theories would look like when axiomatized under a different set theoretical framework, and this could have interesting philosophical consequences. Recently, a paper dealing with first-order relativity theory (both special and general) was posted in the web [1]. Do these versions agree with the 'full' theories of relativity, whose structures are not order-1 structures? In our opinion, the versions the authors obtain of such theories are their first-order versions, and a question to be discussed would be in what sense these formulations fit the original theories, so as if something is left out. The case resembles Tarski's Elementary Geometry, which is not euclidian geometry, and the theory of real closed fields, which is not the full theory of the reals.

The poweful of Suppes's approach Despite its great simplicity, Suppes approach is very powerful indeed, and here we point to another of its advantages. While the standard group axioms (to keep with our example) characterize all groups as being models of the axiomatics, in using set-theoretical predicates we can axiomatize certain classes of models only. A typical example would be to axiomatize all groups except a certain specific class; for simplicity, suppose that we wish to leave out the additive group of the integers, $\langle\mathbb{Z},+\rangle$. We can do it by adding to the set-theoretical predicate given above (see at page 13) a fourth condition $A 4$ (another axiom), namely, a clause restricting the items that satisfy the predicate, say by requiring that those structures that satisfy it are groups but different from $\langle\mathbb{Z},+\rangle$ (this can be easily expressed, say by requiring 
in the set-theoretical predicate that $G \neq \mathbb{Z}$ ). For another example, one can take the Peano structures $\mathfrak{P}=\langle\omega, 0, \sigma\rangle$, with the usual Peano axioms in ZFC. Now, the structure $2 \mathfrak{P}=\langle 2 \omega, 0,2 \sigma\rangle$, where $2 \omega$ is the set of even natural numbers and $2 \sigma$ is the addition of 2 , is also a model of these axioms. One can make a Suppes predicate with the Peano postulates plus the requirement that the domain is different from $2 \omega$, for example, and then this structure is not in the class of models of the set-theoretical postulate for arithmetics. This is possible because in the language of ZFC we can make reference to a particular element we leave outside the class of structures determined by the predicate. This fact is precisely what differs the Suppes approach from da Costa-Chuaqui, and even from Bourbaki. In the last two cases, the predicate (or the axioms in the case of Bourbaki) has two main parts: a typification and the axioms themselves. The typification is a formula, or a conjunction of formulas that specify what are the particular relations/operations/distinguished elements we are considering (for the formal definition, see $[2, \mathrm{p} .261])$. Let us exemplify: in the case of groups, the typification is $\circ \in \mathcal{P}(G \times G \times G)$. In the case of vector spaces, we have $+\in \mathcal{P}(V \times V \times V) \wedge \cdot \in \mathcal{P}(K \times V \times V)$. The axioms, in both cases, must be transportable formulas; roughly speaking, this means that the definition of the formula does not depend upon any specific property of the construction made from the basic and auxiliary sets, but only refers to the way in which they enter in the relation through the axioms [2, loc.cit.]. In the Suppes approach, this restriction is not imposed, that is, the formulas needn't be transportable, and so we gain a wide range of extra possibilities.

For another example of these possibilities, let us consider a simple predicate for a structure composed by a set $D$ and an operation $\sigma$ picked from $t(\langle i, i\rangle)$ (that is, a binary operation on $D$ ) satisfying the conditions (axioms): (a) $\emptyset \in D$; (b) $\sigma$ is injective; (c) $\emptyset$ does not belong to the image of $\sigma$; (d) $D$ is the least set satisfying these conditions. In this case, we are mentioning a particular item, $\emptyset$, and saying in the axioms that this chosen element must belong to the domain of the structure. Since we are making reference to a well defined object (the set $\emptyset)$, the axioms are not transportable formulas. In this particular example, we still have that it is not possible to axiomatize $\mathfrak{M}=\langle D, \sigma\rangle$ in the standard way, say by fixing a specific vocabulary and making use of first-order logic (more on this below).

The fact we can do such moves show that set-theoretical predicates differ from Suppes predicates as defined by da Costa and Chuaqui, so as from Bourbaki's species of structures, although some predicates can fit the claims of transportability, as mentioned in section 4 . The reason is that an axiomatics (say, in Bourbaki's style) should not make reference to specific objects such as the empty set. In fact, Bourbaki requires that the theory's postulates must be transportable formulas with respect to some typification, which teach us how to deal with the added symbols of the structure (see definition 7.4). Saying with other words (in the next section we shall discuss this topic again), transportable formulas must be invariant by isomorphisms, and so they cannot make reference 
to specific objects [2, p.261-2]). ${ }^{10}$ These difficulties can be surpassed with some modifications and adaptations of our example, but they shall not concern us here. Anyway, in the Suppes' style axiomatization, we have free access to all mathematics that can be found in ZFC, without constraints on the kinds of formulas that can be used to axiomatize a theory.

This previous discussion can be summarized in an interesting result. Recall that, when we restrict ourselves to first-order languages, in order to axiomatize a class of order- 1 structures we must give a set $\Gamma$ of sentences such that all the structures in the class are models (in the sense of Tarski) of $\Gamma$, and all models of $\Gamma$ are in the class. A necessary and sufficient condition for a class of structures to be axiomatized by a set of sentences, is that it must be closed by elementary equivalence and by formation of ultraproducts (again, the definitions do not matter us here). Then for example, in this kind of axiomatization, when we deal with groups and the usual postulates for this theory written in a first-order language, we cannot have $\langle\mathbb{Z},+\rangle$ out of the class of structures if $\langle 2 \mathbb{Z}, 2+\rangle$ is in this class (here, $2 \mathbb{Z}$ denotes the set of even integers, and 2+ addition of 2), for both are elementarily equivalent. The same holds for models of first-order Peano arithmetics, for the standard model $\mathcal{P}=\langle\omega, 0, s\rangle$ and $2 \mathcal{P}=\left\langle 2 \omega, 0, s^{\prime}\right\rangle$, where $2 \omega$ is the set of even numbers and $s^{\prime}$ is the operation of adding 2 . That is, we cannot have an axiomatization in a first-order language of one of them without having the other one as well, for they are elementarily equivalent. But, as we have seen, using the Suppes style axiomatization, we can define classes of models which are order- 1 structures but which are not closed by elementary equivalence. That is, Suppes' axiomatization is stronger than the usual one, since it axiomatizes more classes of structures than it is possible to do with the usual procedure, and for checking this we need only to consider the examples given above. So, the examples above show that

Theorem 6.1 There are classes of order-1 structures axiomatizable by a Suppes predicate but not by a set of first-order set of sentences.

This theorem can be generalized to higher-order languages by following the same procedure (just take the class of all well-orderings except a particular well-ordering, for instance $\langle\omega, \in\rangle)$.

\section{Theories as models: part II}

In [4], da Costa and Chuaqui proposed a formalization of Suppes' ideas. We recall some of their definitions here, and discuss their version of what they have called 'Suppes predicate'. As a result, it will soon appear that their formalization make a detour through formalized languages, and so some conditions must be imposed on the formulation of axioms; they must be transportable formulas.

\footnotetext{
${ }^{10}$ We thank Antonio M. N. Coelho and Newton C. A da Costa for pointing us these points and for discussions on these themes.
} 
This feature of the axiomatization by da Costa and Chuaqui is directly influenced by the Bourbakist approach; really, they say that the two approaches are equivent. These topics will be addressed in due time.

We have seen before what is the language of a structure (page 5). Thus, to every structure $\mathfrak{A}=\left\langle D, R_{\iota}\right\rangle$ we can canonically associate to it a language $£(R)$ of the same signature of the structure. Since we are supposing that our structures are not only order1, we may suppose that the following is given to cope with $£(R)$ :

(a) For each type $a \in \tau$, a denumerable set of variables of that type;

(b) Sentential connectives: $\neg$ (not), $\rightarrow$ (implies), $\wedge$ (and), $\vee$ (inclusive disjunction);

(c) For every type $a$, an identity relation $={ }_{a}$ for that type;

(d) Quantifiers $\forall$ and $\exists$;

(e) For every relation in $\mathfrak{A}$, a relation symbol of the same type as the considered relation.

We define $\leftrightarrow$ as usual. As it is easy to see, if we enable $a$ vary over the whole set $\tau$ of types, this schema gives us a language suitable for Simple Theory of Types (STT). The definition of formula is the usual one, respecting the types restrictions. A first point to be mentioned concerning languages associated with structures is that we do not always need the full power of STT; that is, there are many situations in which less powerful languages can be employed, for example $n^{\text {th }}$-order languages or even first-order languages, but the language of theory of types is useful when some considerations come into play, as investigations on definability require (see [5]). This is one the main features of this kind of approach, that is, different languages can be employed and the consequences of doing so can be investigated.

Given the above schema, we can introduce the usual semantical concepts, like semantical consequence, and semantical validity. Notice that we deal only with principal models, that is, we are assuming that the variables of type $a$ have the whole set $t(a)$ as their range, and then the so-called Henkin models for higher order structures are not being discussed here (this is another point where this kind of approach starts distancing itself from the one presented above, that is, here we have the possibility that we could allow also Henkin models in our investigations and, then, we would have the usual distinction between primary and secondary models, a distinction absent in the first kind of axiomatization we saw above). Obviously, Henkin models are obtained when we allow only a subset of $t(a)$ as range of the variables of type $a$, and when the relations of the structure are also members of these selected subsets.

A second point to be noticed is that we could in principle allow our language to be infinitary, with conjunctions and disjunctions of some specified infinite length (this is also relevant for investigations on the concept of definability, see $[5])$. For example, in an infinitary language for a structure $\mathfrak{A}$ with cardinality 
$\kappa_{D}$, besides the symbols presented above, we fix some cardinal $\kappa \leq \kappa_{D}$, and then introduce two connectives $\bigwedge$ and $\bigvee$, that are used for conjunctions and disjunctions of families of formulas whose domains are ordinals strictly less than $\kappa$. The canonical interpretation in $\mathfrak{A}$ is also as usual, and the standard semantic notions can also be defined. In both finitary and infinitary languages, we suppose that the sequences of quantifiers in a formula are always of finite length.

Given a structure and a language $£(R)$ for this structure, we now discuss how to formulate a formalized version of Suppes set-theoretical predicate for this structure using this language in the same vein as proposed by da Costa and Chuaqui [4]. As a first step, we introduce the definition of similarity type of a structure $\mathfrak{A}$, which is a family of types that determines the kinds of the relations present in the structure.

Definition 7.1 The similarity type of $\mathfrak{A}=\left\langle D, R_{\iota}\right\rangle$ is an ordered family $s_{\lambda}$ of types, such that for each $\lambda<\iota, s_{\lambda}$ is the type of $R_{\lambda}$.

That is, the similarity type of a structure determines the types of the relations of a structure and the order in which they appear. According to this definition, two structures have the same similarity type if the types of their relations are the very same family.

Now, given two structures $\mathfrak{A}=\left\langle D, R_{\iota}\right\rangle$ and $\mathfrak{B}=\left\langle E, L_{\iota}\right\rangle$ of the same similarity type, we consider how to extend a given function $f: D \mapsto E$ to a mapping from $\varepsilon(D)$ to $\varepsilon(E)$ (see $[4])$.

Definition 7.2 Given a function $f$ as described above, for each type $a \in \tau$ we define:

(i) for the objects of type i, $f(D)=\{f(x): x \in D\}$;

(ii) for $a \in \tau$ such that $a=\left\langle a_{0}, \ldots, a_{n-1}\right\rangle$, and $R$ the set of objects of type a, we have $f(R)=\mathcal{P}\left(f\left(t_{a_{0}}\right) \times f\left(t_{a_{1}}\right) \times \cdots \times f\left(t_{a_{n-1}}\right)\right)$

This function maps objects of the type $a$ in $\varepsilon(D)$ to objects of type $a$ in $\varepsilon(E)$. We need to consider the case when the following further condition is verified:

Definition 7.3 Given two structures $\mathfrak{A}=\left\langle D, R_{\iota}\right\rangle$ and $\mathfrak{B}=\left\langle E, L_{\iota}\right\rangle$ of the same similarity type $s_{\lambda}, \lambda<\iota$, let $f$ be a bijection from $D$ to $E$. We say that the family $f^{\prime}=f_{s_{\lambda}}$ is an isomorphism between $\mathfrak{A}$ and $\mathfrak{B}$ when $f_{a}\left(R^{a}\right)=L^{a}$, where $R^{a}$ and $L^{a}$ means that $R$ and $L$ have type $a$.

Definition 7.4 A sentence $\Phi$ of the language of the structure $\mathfrak{A}$ is called transportable if for any structure $\mathfrak{B}$ isomorphic to $\mathfrak{A}$ we have

$$
\mathfrak{A} \models \Phi \Leftrightarrow \mathfrak{B}=\Phi
$$

Definition 7.5 A Suppes'predicate is a formula $\mathrm{P}(\mathfrak{A})$ of set theory which says that $\mathfrak{A}$ is a structure of similarity type s satisfying $\Gamma$, which is a set of transportable sentences $\Phi$ of the language adequate for $\mathfrak{A}$. 
When $\mathrm{P}(\mathfrak{A})$, that is, when $\mathfrak{A}$ satisfies $\mathrm{P}$, we say that $\mathfrak{A}$ is a $\mathrm{P}$-structure. According to da Costa and Chuaqui [4, p.104], this definition captures the sense in which we can say that a theory is a class of models, precisely, the class of models that are P-structures for some adequate P. Now, these structures are really models of the set of sentence comprised in $\Gamma$ in the logicians sense, so here we have a set of sentences that are satisfied by some structure.

The first point to be mentioned concerning this version of axiomatization is that it employs formal languages (built within ZFC) in the formulation of the axioms. As we mentioned before, one does not always need to employ the full resources of theory of types. It is an interesting question to investigate whether a class of models can be determined by axioms written in some kind of language but not in other languages. It would also be interesting to investigate what are the effects of restricting oneself to languages of some specific order. So, for example, for the predicate for arithmetic, if we employ first-order language in the formulation of the axioms, we allow that non-standard models enter in the class of models. If we employ second or higher order languages, such models are no longer available, and the axiomatized theory is in general categorical. Also, infinitary languages are interesting when many questions about definability come into play, and so, a huge amount of philosophical work can be done on a rigorous basis.

It seems that one interesting point for the philosophers of science is the specification (if possible) under which conditions the theories' specific axioms could be written exclusively in first-order languages. This is a very special kind of language, and some claim it has distinguished clarity. This would be important because in using first-order languages, it would be possible to employ model theoretic tools when restricted to these languages (recall that there is no fully developed model theory for higher order languages). But we should proceed with care for as we have already mentioned, there is a distinction between a theory axiomatized in its 'full' version, say by using higher-order resources, and a first-order version of it (we have made reference to the first-order versions of relativity theory, elementary geometry and real closed fields before). To recall another important point, we notice that there are some discussions on whether first or second order languages are more appropriate for mathematical discourse (see, for example, [17]).

On the employed languages Let us speak a little bit more about this last topic. It really makes a difference whether we use first or higher order languages in our axiomatization of, say, arithmetics. In the first case, we have non-standard models, and the theory is not categorical while, in the second case, the models are all isomorphic. This is an interesting point, and we think that our distinction between the two kinds of axiomatizations presented in this work may help us in putting some order in the discussion. One first argument in favor of first order languages is that in the mathematical practice we always treat higher order structures with first order languages; we have already seen that well-ordered structures and Dedekind-complete fields are not order-1 struc- 
tures. Although they cannot be formalized as first-order theories, we can deal with them within first-order ZFC. This distinction is important, let us insist. Within first-order ZFC we can build higher-order structures. This is true, but one must notice that in this case these structures are being treated according to the first approach presented here (the Suppes approach), in which the axioms are written in the language of set theory proper. If first order languages are employed, according to the second semantic approach (in the sense of da Costa and Chuaqui), we would not get the required expressive power to characterize these higher-order structures (in this case, second or higher order languages would be more adequate). If the debate is meant to be conducted inside our second (semantical) approach to structures, then there will be significant differences when we adopt only first-order languages or also higher order languages, but we see no reason why only one of the options should be preferred, and both should be investigated. This shows the great power of the language of first-order set theory.

The distinction between the orders of languages and their influence on the class of models that is being characterized is another topic which would deserve further investigation by philosophers. This is another aspect of the discussion mentioned in the last paragraph. Let us mention only some of the involved questions, which we intend to investigate in a future work. A further position, different from those already mentioned, attributes little real importance for the whole debate, for it would be ill motivated (this is roughly the position expressed in Hodges [9, pp. 71-73]). The main basis for this assertion is a well known procedure according to which it is possible to translate sentences of a higherorder language to a convenient polyadic first-order language with a kind of membership relation (see[5, pp.8-9] for the definition of the general procedure). For example, for a second order language, we introduce two kinds of variables. Of the first kind are the variables for individuals, while second-order variables for relations and properties are of the second kine. A membership relation is also introduced. It relates variables of the first kind to variables of the second kind, that is, statements that in a second order language were a form of predication, now become membership. This kind of argument seems framed in our second approach to theories. So, could we say that it really does not matter what kind of language we employ in characterizing a class of models? But, as we mentioned, there is some difference in first and second order arithmetics, for example. So, what is really at stake here? The point is that we cannot simply translate all higher order theories to first order theories and think that firstorder languages are all that is needed; what needs to be mentioned in the first place is that, translating, for example, second order arithmetics into a firstorder theory, does not gives us the usual first-order theory, but a sophisticated version with different sorts of variables and further complications, like a kind of membership relation (this completes what we have said about the first-order versions of relativity). Also, it must be noticed that this move from higherorder to first-order theories is always relative to a structure: given a structure of higher-order, we can find an associated first-order structure and, if working in this first-order structure some interesting result is found, we can then look back 
to the higher-order structure and associated language in order to re-interpret the result (this 'technique' is shown in [5], where it was used for applications in problems of definability, but that we think that it should be considered for more general applications). So, it is not easy to see how to justify the claim that the distinction between orders of languages are blurred when we restrict ourselves to specific theories. The point is that, given a higher-order structure, we can always work in a complicated but first-order structure associated with it, and the results can be the taken back to the higher-order structure (ibid.). As we mentioned before, we regard as interesting and important to study the consequences for axiomatization of classes of models when different languages are employed. This kind of consideration may have interesting consequences when empirical theories are considered, for example, the possibilities of nonstandard models when first-order languages are used, but we shall leave this kind of problem for another work.

\section{Suppes, Muller, and ourselves}

We have discussed above that Suppes style axiomatization is stronger than the usual one. There is another point in which we can see that both approaches differ. As Muller pointed out in section 6 of his [16], in Suppes approach the structures that characterize a scientific theory are not models in the Tarskian sense, for, as we have discussed, among other things there is not a formal language being interpreted, and no definition of truth and semantical consequence relation being employed. Differently from us, Muller goes on to conclude from this that Suppes approach is not really a semantical approach (ans Suppes agrees, as we have said). We shall not dispute this claim here, for we are interested in a tolerant definition of what the semantic approach amounts to, and also in Muller's following question: how can we complete the Suppesian approach, in order that it uses models in the Tarskian sense? To do that, Muller notices, we must provide a formal language and axioms in this language for our classes of structures, which will be the models of the axioms, now in the Tarskian sense, precisely as in the da Costa-Chuaqui style. This move, even though it determines a class of models, has an undesirable drawback: the non-logical vocabulary determines the type of similarity of the structures that model sentences in this language, and then only structures of that similarity are collected by a particular axiomatization. To consider an example, the vocabulary for groups can be taken to be $£_{G 1}=\{0\}$, with a binary operation only, or alternatively, $£_{G 2}=\left\{0, e,^{-1}\right\}$, including, besides the binary operation, the null element and the inverse operation. Now, the problem - as Muller sees it - is that despite the fact that the theory axiomatized using these different vocabularies is in both cases 'the same'

theory, the classes of structures modeling each formulation will be different, for they are of different types of similarity. So, how can a theory be seen as a class of structures? Do we have two group theories? Muller's proposal to overcome this difficulty is roughly as follows: one uses as axioms the sets of sentences that are translatable one into another, so that, despite having different types of 
similarity, structures in the class satisfy at least one of the possible formulations of the axioms. This move is done in order to define a class of models that can be called somehow 'language free', in the sense that the language does not matter to determine the class of structures, but still considers the class of structures as a class of models in the Tarskian sense. Sure, the employed language matters, otherwise there would be not the necessity of this trick to overcome its effect over the class of selected structures. But, according to Muller, the idea is that the particular language employed is not essential, whatever that might mean.

We thing this idea an interesting one, but there are two difficulties associated with it, one from a pragmatical point of view, and the other from a more technical point of view. From the pragmatical side, it seems that this approach leaves behind the idea that philosophy of science should employ mathematics, not metamathematics. In fact, the whole idea of languages and translations among them is done in metamathematics, and so, this does not seem a good move for improving the Suppes approach (as Muller claims). Suppes approach is based on usual mathematical practice, and does not rely on these issues, and chooses, according to pragmatic principles, which kind of structure to adopt in each case, according to convenience. For example, in the studies of groups, it is simpler to take $£_{G 1}$, for it is easier to deal with it, but sometimes, when we are specially interested in subgroups, $£_{G 2}$ is better, for every substructure of a model of the axioms formulated in this vocabulary will also be a subgroup, something that does not follow in the first case. For the second objection, we recall the theorem 6.1, according to which the Suppesian approach is stronger than the usual one, in the precise sense that some classes of structures are axiomatizable only with these tools, and not in the usual sense, to which Muller wants to return. So, it is not clear that Muller's proposal is not really an improvement for, although he reduces the first approach (Suppes) to the second (da Costa-Chuaqui), we loose in strength and in simplicity. Obviously, since we are advocating that both approaches deserve further study, this does not mean that one of them should be favored over the another, but only that, as a fact of life, the first is stronger and simpler to use.

\section{$9 \quad$ Final Remarks}

Within the semantic approach we have discussed two distinct directions in the axiomatization of scientific theories: one following more directly Suppes, in which a predicate is written in the language of set theory itself, and another, following da Costa and Chuaqui, in which formal languages are employed to write the specific postulates of the theory we wish to axiomatize. Both approaches can be employed to specify a class of structures which can, in different ways, be called the models of the theory. So, both approaches can be said to underpin some version of the so-called semantic approach to scientific theories. Thus, what is the semantic approach about? Well, it depends on the way the set-theoretical predicate is being considered. Obviously, still further versions which do not involve the formulation of a set-theoretical predicate can be con- 
ceived in axiomatizing scientific theories (and mathematical theories as well), but the approach by set-theoretical predicates, which can be understood as stating the theory's specific postulates, in order to gather somehow the models of it, is the most well-known. We have also argued that it is not always the case that models are taken in the standard Tarskian sense, and that this is simply a phenomena following from the ambiguity there exists in specifying precisely what a semantic approach is (an, after all, what is really semantical in the semantic approach). Furthermore, we have also enlighten that language matters even for the semantic approach, so the usual reference that it is concerned with models only, should be taken with some care; in fact, different languages are used in the different ways that can be used to axiomatize a theory. If one writes the theory's postulates 'directly' with a set theoretical language, then the first proposal mentioned here is used (Suppes); on the other hand, if we consider the use of formal languages to state the postulates, then the second proposal can be used (da Costa and Chuaqui), and there will be relevant matters on which a certain kind of language is more suited for the work we wish to do. As we have argued, each approach have some benefits and costs, which will have some consequences when the philosophical work on the models and the theories they characterize step into the stage.

\section{References}

[1] Andréka, H., Madarász, J. X., Németi, I. and Székely, G. , A logic road from special relativity to general relativity, http://arxiv.org/abs/1005.0960

[2] Bourbaki, N., Theory of Sets, Hermann \& Addicon Wesley.

[3] Brignole, D. and da Costa, N. C. A., On supernormal Ehresmann-Dedecker universes, Mathematische Zeitschrift 122, 1971, 342-350.

[4] da Costa, N. C. A. and Chuaqui, R., On Suppes' Set Theoretical Predicates, Erkenntnis, 29: 95-112, 1988.

[5] da Costa, N. C. A. and Rodrigues, A. A. M., Definability and Invariance, Studia Logica, 82: 1-30, 2007.

[6] da Costa, N. C. A. and Krause, D., Physics, inconsistency, and quasi-truth, forthcoming.

[7] da Costa, N. C. A., Krause, D. and Bueno, O., Paraconsistent logics and paraconsistency, in D. Jacquette (ed.) Philosophy of Logic, Vol.5 of D.M.Gabbay, P.Thagard and J.Woods (eds.), Handbook of the Philosophy of Science, 2007, pp. 655-781.

[8] French, S. and Krause, D., Remarks on the theory of quasi-sets, Studia Logica, 2010, Online. 
[9] Hodges, W., Elementary Predicate Logic, in Gabbay, Dov. M., and Guenthner, F., (eds.) Handbook of Philosophical Logic, vol. 1, 2nd. edition, 2001, Springer.

[10] Kaye, R., Models of Peano Arithmetic, Oxford, Clarendon Press, 1991.

[11] Kunen, K., The Foundations of Mathematics, College Publications, London, 2009.

[12] Ludwig, G., An Axiomatic Basis for Quantum Mechanics, Vol. I, SpringerVerlag, 1985.

[13] Maitland Wright, J. D., All operators on a Hilbert space are bounded, Bulletin of the American Mathematical Society 79 (6), 1973, pp.1247-1250.

[14] McKinsey, J. C. C., Sugar, A. C. and Suppes, P. 1953, Axiomatic Foundations of Classical Particle Mechanics. Journal of Rational Mechanics and Analysis 2 (2),1953, 253-272.

[15] Moore, G. H., Beyond first-order logic: the historical interplay between mathematical logic and axiomatic set theory, History and Philosophy of Logic 1, 1980, 95-137.

[16] Muller, F. A., Reflections on the revolution at Stanford, Synthese, DOI: 10.1007/s11229-009-9669-7.

[17] Shapiro, S., Foundations without foundationalism, a case for second-order logic, Clarendon Press, Oxford, 1991.

[18] Suppe, F. (ed.), The Structure of Scientific Theories, Urbana \& Chigago, Chicago Un. Press, 2a. ed., 1977.

[19] Suppes, P., Introduction to Logic, New York:van Nostrand, 1957.

[20] Suppes, T., Representation and Invariance of Scientific Structures, Stanford, Center for the Study of Language and Information, 2002.

[21] Suppes, P., Future developments of scientific structures closer to experiments: Response to F. A. Muller, Synthese, online, DOI: 10.1007/s11229009-9670-1. 